\title{
Spectroscopy of spin-polarized excitons in semiconductors
}

\author{
E.L. Ivchenko \\ A.F.Ioffe Physico-Techrical Institute, Politechnicheskaya 28, 194021 St.Petersburg, Russia
}

\begin{abstract}
Excitons in semiconductora can be spin-polarized under optical selective excitation by polarized radiation (optical orientation) or due to apin relaxation and anblevel mixing in an external magnetic field. The paper deals with optical phenomena where spin-polarized excitons generated in semiconductor nanostructures play an important role. Firstly, both optical orientation and optical alignment of excitons in type II GaAs/AlAs superlattices are considered and effects of the arisotropic electron-hole exchange interaction and external magnetic field on the photoluminescence polarization are analyzed. Secondly, magnetic-field-induced anticrossing of excitonic sublevels is liscussed taking into account the axial and anisotropic exchange splittings, spin-relaxation and difference in the lifetimes of radiative and non-radiative exciton states. Next, the localized and bound excitons are shown to act as intermediate atates in resonsnt Raman scattering by apin flips of holes bound to acceptors in GaAs/AlGaAs maltiple quantum wells. The analysis of polarized Raman spectra permits to make decisive conclusions concerning microscopic mechanisms of the observed scattering prosesses. Finally, the donbly-resonant 2a-1s LO-assisted secondary emission observed in $\mathrm{CdTe} / \mathrm{CdMnTe}$ quantum-well structures is described as a process with spin-polarized hot 1s-excitons acting as real intermediate states.
\end{abstract}

\section{INTRODUCTION}

The exciton spin polarization in bulk semiconductors was extensively studied in 70 -ies and early 80 -ies (see the review ${ }^{1}$ and references therein). In the absence of an external magnetic field spin-polarized excitons can be generated under optical pumping with circularly polarized photons: due to spin-orbit coupling of electronic states the selection rules for optical interband transitions provide a conversion of photon polarization into photocarrier or exciton apin orientation. In accordance with the selection rales the radiative recombination of spin-polarized excitons results in the circular polarization of the photoluminescence thus making possible optical detection of the spin polarization. The optical orientation of excitonic spins is a particular case of the more general phenomenon, namely, the selective optical excitation of excitonic sablevels. Another example of the selective excitation is the so-called optical alignment of excitons by linearly polarized radiation: in contrast to the optical orientation which means just the photoinduced difference in the populations of the exciton states $|m\rangle$ with the spin $m= \pm 1$, the linearly-polarized light can excite preferentially the exciton state $\left(\left|+1>+e^{i \phi}\right|-1>\right) / \sqrt{2}$ with a definite direction of oscillating electric-dipole moment (a value of the phase $\Phi$ is determined by the direction of the light polarization plune). Application of an external field (magnetic field, strain) reduces symmetry of the system and can lead to interconnection between exciton optical orientation and alignment in which case it is necessary to consider not only circular-circular and linear-linear but also mixed circular-linear or linear-circular configurations of the polarizer and analyzer.

The optical orientation effect can be readily observed if the spin relaxation time, $T_{d}$, is not too small relative to the exciton lifetime, $\tau$. If however $\tau_{1}<\tau$ a more convenient method is to observe the thermal orientation of spins in a magnetic field: due to apin relaxation upper and lower aplit sublevels become selectively populated even under anpolarized excitation. There exist also non-thermal mechanisms of exciton spin polarization under unpolarized excitation, they are connected with magnetic-field-induced mixing and anticrossing (see e.g. ${ }^{2}$ ) of excitonic sublevels.

The present paper touches various aspects of the exciton spin polarization in semiconductor nanostructures. Optical selective excitation of excitonic sublevels in type II superlattices is considered in Section 2. The results on optically-detected exciton level-anticrosaing in an external magnetic field are presented in Section 3. Bonnd and localized spin-polarized excitons acting as intermediate states in resonant Raman spin-lip scattering processes are analyzed in Section 4. Section 5 is devoted to optical orientation of hot excitons and their involvement in the doubly-resonant $2 s-1 s$ secondary emiesion observed recently in $\mathrm{CdTe} / \mathrm{Cd}_{1-s} \mathrm{Mn}_{x}$ Te quantum well structures ${ }^{3}$.

\section{OPTICAL ORIENTATION AND ALIGNMENT OF EXCITONS IN GaAs/AlAs SUPERLATTICES}

We show the efficiency of optical selective excitation methods on GaAs/AlAs(001) superlattices which are a convenient model object to study type I - type II transitions in heterostructures. Really, depending on the layer thicknesses the bottom of the conduction band in these structures is formed mainly either from states of the $\Gamma$. minimum in the GaAs layer (type I) or from states of the $X$-minimum in the AlAs layer (type II). The lowest excitonic level $e 1-h h 1(1 s)$ in both types consists of four sublevels taking into account the electron $( \pm 1 / 2)$ and heavy hole $( \pm 3 / 2)$ spin degeneracy. Symmetry considerations show that, in an ideal structure, due to the electron-hole exchange interaction the exciton state is split into a radiative doublet and two close-lying non-radiative sublevels. 
However experimente on polarized luminescence resonantly excited by circularly or linearly polarized light $t^{4,5}$ and on optically detected magnetic resonance ${ }^{b}$ showed that, for localized excitons in type II GaAs/AlAs soperlattices, (i) the degeneracy of the radiative doublet is lifted, (ii) the two split sublevels are dipole-active along the [110] and [1I0] directions in the layer plane, and (iii) in a single superiattice with fixed layer thicknesses two classes of excitons can be excited simultaneonsly with the same value but opposite signs of the anisotropic exchange splitting. In ${ }^{7}$ we have explained the nature of this giant anisotropic splitting taking into account the mixing of heavy and light hole states on the interfaces and connecting the two classes of localized excitons with those attached to the GaAs/AlAs and AlAs/GaAs interfaces. The recent numerical calculations have confirmed the proposed idea about considerable heavy-light hole mixing by the (001) inteface at normal incidence of the hole.

Optical orientation and alignment of excitons can be described by noing the density matrix formalism ${ }^{1,5,9}$. In a sterdy-atate regime of photoexcitation, the components $\rho_{j j^{\prime}}$ of the localized-exciton density matrix satiofy the binetic equation

$$
\left(\frac{\partial \rho}{\partial t}\right)_{\text {reo }}+\left(\frac{\partial \rho}{\partial t}\right)_{\text {i.r. }}+\frac{i}{\hbar}\left[\rho, \mathcal{K}_{\text {eech }}+\mathcal{K}_{\mathrm{B}}\right]=G .
$$

The terms on the left-hand aide take account of the exciton recombination, spin relaxation, exchange and Zeeman interaction in the presence of magnetic field $\mathbf{B}, G$ is the generation matrix. Let $|m\rangle_{0}$ be the basis atates of the $e 1-h h 1(1 s)$ exciton with the angular momentum component $m=s_{3}+J_{1}= \pm 1, \pm 2$. In this basis the exchange term in the spin Hamiltonian is given by

$$
\mathcal{H}_{\text {o* oh }}=\frac{1}{2}\left[\begin{array}{cccc}
\delta_{0} & i \delta_{2} & 0 & 0 \\
-i \delta_{2} & \delta_{0} & 0 & 0 \\
0 & 0 & -\delta_{0} & \delta_{1} \\
0 & 0 & \delta_{1} & -\delta_{0}
\end{array}\right],
$$

where $\delta_{n}$ are the exchange interaction constants ${ }^{7}$ with $\delta_{2}$ describing the above-mentioned anisotropic exchange splitting. The recombimation term has a standart form

$$
\frac{\partial \rho_{m m^{\prime}}}{\partial t}=-\left(\frac{1}{\tau_{m}}+\frac{1}{\tau_{m^{\prime}}}\right) \rho_{m m^{\prime}}, \frac{1}{\tau_{m}}=\frac{1}{\tau_{f}} \delta_{|m|, 1}+\frac{1}{\tau_{0}}
$$

where $\delta_{j, j}$ is the Kronecker symbal, $\tau_{r}$ is the radiative lifetime for the exciton states $| \pm 1\rangle_{0}, \tau_{0}^{-1}$ is the non-radiative recombination rate which is usually assiamed to be the same for all states.

Neglecting for simplicity the exciton epin relaxation we obtain for the photoluminescence circular polarization under resonant circularly-polarized excitation in the longitudinal magnetic field

$$
P_{0}(\mathbf{B} \| z)=P_{c}^{0} \frac{1+\Omega_{\|}^{2} \tau^{2}}{1+\left(\Omega_{\|}^{2}+\tilde{\omega}^{2}\right) \tau^{2}}
$$

where $z$ is the growth direction of the structure, $\tilde{\omega}=\delta_{2} / \hbar, \hbar \Omega_{\|}=\left(3 g_{h}-g_{b}^{\|}\right) \mu_{0} B_{n}, g_{l}^{\|}$is the electron longitudinal $\mathrm{g}$-factor, $g_{h}$ is the heavy-hole $g$-factor defined so as the hole Zeeman energies are given by $\pm 3 / 2 g_{h} \mu_{0} B, \mu_{0}$ is the Bohr magneton, $\tau \equiv \tau_{ \pm 1}, P_{0}^{0}$ is the circular polarization degree of the incident light. In type II GaAs/AlAs superiattices the anisotropic splitting exceeds the value of $\hbar / \tau$ so that $(\bar{\omega} \tau)^{2}>1$ and at zero magnetic field the motoluminescence is practically unpolarized. The longitudinal magnetic field aupresses the depolarizing eifect of anisotropic exchange interaction and permits to observe the optical orientation of excitons (see Fig.1).

Fig. 1 Restoration of circular polarization of the el - hhl exciton luminescence in the presence of the longitudinal magnetic field. Crosses are experimental points, solid curve is calculated by using equation $P_{0} \propto 1-\left[1+\left(B / B_{1 / 2}\right)^{2}\right]^{-1}$ with $B_{1 / 2}=0.25 \mathrm{~T}^{3}$

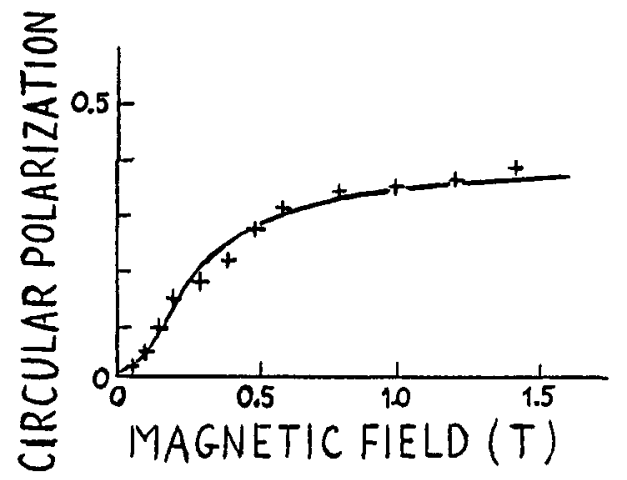


Eq.(4) describes relative radiation intensities only for circular-circular polarization configurations. A complete set of relations between polarizations of the incident and secondary radiations can be presented in the following form

$$
\begin{gathered}
P_{c}=\frac{\left(1+\Omega_{\|}^{2} \tau^{2}\right) P_{c}^{0}-\Omega_{\|} \tilde{\omega} \tau^{2} P_{l}^{0}+\tilde{\omega} \tau P_{\|}^{0}}{1+\left(\Omega_{\|}^{2}+\tilde{\omega}^{2}\right) \tau^{2}} \\
R_{1}=\frac{-\tilde{\omega} \Omega_{\|} \tau^{2} P_{c}^{0}+\left(1+\tilde{\omega}^{2} \tau^{2}\right) P_{l}^{0}+\Omega_{\|} \tau P_{l}^{0}}{1+\left(\Omega_{\|}^{2}+\tilde{\omega}^{2}\right) \tau^{2}} \\
A_{1}=\frac{-\tilde{\omega} \tau P_{c}^{0}-\Omega_{\|} \tau P_{l}^{0}+P_{i}^{0}}{1+\left(\Omega_{\|}^{2}+\tilde{\omega}^{2}\right) \tau^{2}}
\end{gathered}
$$

Here $A$ and $A_{1}$ are the degrees of linear polarization referred respectively to the rectangular axes $x\|[1 \overline{10} 0], y\|[110]$ and $x^{\prime}\left\|[100], y^{\prime}\right\|[010]$ :

$$
A=\frac{I_{x}-I_{y}}{I_{x}+I_{y}}, R_{11}=\frac{I_{x}^{\prime}-I_{y}^{\prime}}{I_{z}^{\prime}+I_{y}^{\prime}} .
$$

the superscript 0 indicates polarization of the incident light.

The following additional conclusions can be made from the analysis of Eqs.(5-7): (1) For large anisotropic splitting, $\tilde{\omega} \gg \tau^{-1}$, the polarization $A_{1}$ is negligibly small irrespective to the incident light polarization and magnetic field value. (2) At zero field, the excitons can be aligned under optical pumping in the polarization $E \| x$ or $y$ in agreement with the above-mentioned fact that the split radiative sablevels are polarized along the $[1 \overline{1} 0)$ and [110] directions. (3) The magnetic-field-induced depolarization of optical alignment is described by the Lorentzr type curve with the halfwidth $B_{1 / 3}=\left|\delta_{3} /\left(3 g_{h}-g_{\mathrm{l}}^{\|}\right) \mu_{0}\right|$ which also governs the restoration of $P_{c}$. (4) Eqs.(5-7) give the polarization as a function of the magnetic field $B_{3}$ for excitons with fixed sign of $\bar{w}$. If excitons of both classes are taken into consideration the $\tilde{\omega}$-odd polarization degrees should be reduced because of the compensation. In the absence of complete compensation, i.e. if the densities of states for the two classes of localized excitons differ from each other, the circular-to-linear or linear-to-circular conversion can take place with the effect being maximal at $B=B_{1 / 2}$. The spin relaxation processes modify the $6 \times 6$ matrix connecting $P_{c}, P_{1} P_{1}$ with $P_{c}^{0}, P_{1}^{0}, P_{i l}^{0}$ but anyhow the main conclusions hold valid.

The time-resolved polarized photolumineacence can be also analyzed in the frame of the density-matrix formalism by adding to the leit-hand side of Eq.(1) the time derivative $d \rho / d t$. Due to the anisotropic splitting between the [110]- and [110]-polarized sablevels the selective excitation of localized excitonic states with a $\langle 100\rangle$ polarized light is followed by polarization quantum beats with the period $T_{b}=2 x \hbar /\left|\delta_{2}\right|$. This enables one to make the most direct measurements of the splitting $\delta_{2}$. The experimental values of $\delta_{2}$ measured by optically detected magnetic resonance and quantum beats techniques are presented in Fig.2 together with the theoretical curves. The similar quantum beats can be excited with a circularly polarized light pulse. Moreover, in the case of two classes of excitons with different populations the circular polarization oscillation coexists with an appearance and $\pi / 2$-phase-shifted oscillation of linear polarization $P_{1}$ in a similar fashion as the circular and linear polarizations coexist in the light wave propagating perpendicularly to the principal axis of a birefringent medium. The transverse magnetic field $B \perp z$ couples the radiative and non-radiative atates in which case two periods are observed in the quanturm beats the long and short periods corresponding to the amall and large splitting energies of the excitonic quartet ${ }^{10}$.

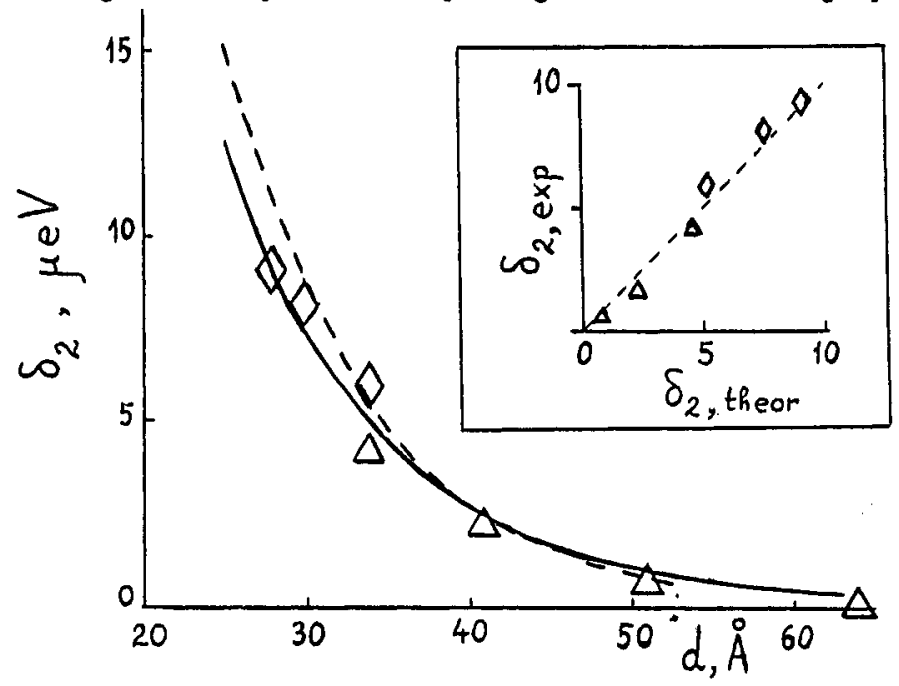

Fig. 2 Anisotropic exchange splitting of the radiative doublet as a function of the GaAs/AlAs superlattice period. Triangles ${ }^{\circ}$ and rhombes ${ }^{4}$ show experimental results, solid and dashed carves are calculated by uing slightly different models?. 


\section{OPTICALLY DETECTED EXCITON LEVEL-ANTICROSSING}

This section responds to the experiment ${ }^{11}$ and extends the theory of optically-detected anticrossing of excitonic levels in the longitudinal magnetic field, $B \| z$, from boll crystals ${ }^{2}$ to anperlattices. The preliminary resulta have been reported in ${ }^{9}$.

The energies of the Zeeman-split quartet are given by

$$
E_{1,2}=\frac{1}{2}\left[\delta_{0} \pm \sqrt{\delta_{2}^{2}+\left(3 g_{h}-g_{t}^{\sharp !}\right)^{2} \mu_{0}^{2} B^{2}}\right], \quad E_{3,4}=\frac{1}{2}\left[-\delta_{0} \pm\left(3 g_{h}+g_{\epsilon}^{\Downarrow}\right) \mu_{0} B\right] .
$$

At zero magnetic field the atates 1,2 are the [110]- and [1 10$]$-polarized excitons, the sublevels 3,4 coincide with the above basis excitonic atates $| \pm 2\rangle_{0}$ since zero-field splitting between them is hereafter neglected $\left(\delta_{1}=0\right)$. At two particular values of $B$ the $E_{3}$ level crosses the levels $E_{1}$ and $E_{2}$. Near the crossing points the statea 1,3 or 2,3 can be strongly mixed even by a weak aymmetry-breaking potential $V$ that couples radiative and non-radiative states. 'This effect known as the level-anticrosaing can lead to a remarkable non-thermal exciton apin polarization under anpolarized excitation. One can set for the matrix elements of this perturbation

$$
V_{m, m^{\prime}}=V_{0} \eta_{m m^{\prime}}, \eta_{m m^{\prime}}=\left.\delta_{\mid m-m^{\prime}}\right|_{1}
$$

which corresponds to the presence of a small transverse magnetic-field component, $\mathbf{B}_{\perp}$. In this case the anticrossing parameter, $V_{0}$, is proportional to the electron transverse g-factor, $g_{0}^{\perp}$, because for heavy holed $g_{h}^{\perp}=0$. Similarly, one can also consider the anticrosaing mechanism dae to atatic diatortions mixing the heavy-hole states $\pm 3 / 2$ in which case $\eta_{m m^{\prime}}=\delta_{\mid m-m} \mid, s$. It is convenient to expand the eigenstates $\left.\mid j\right)(j=1-4)$ of the spin Hamiltonian $\mathcal{H}=\mathcal{H}_{\text {exch }}+\mathcal{H}_{\mathrm{B}}+V$ in terms of the basis states, so that

$$
|j\rangle=\sum_{m} C_{j m}|m\rangle_{0}
$$

In order to calculate the intensity and polarization of the localized exciton photoluminescence we explore the following set of steady-state rate equations for the level populations $f_{j}$ :

$$
\begin{aligned}
& \left(\frac{\partial f_{j}}{\partial t}\right)_{\text {rec }}+\left(\frac{\partial f_{j}}{\partial t}\right)_{\text {o.f. }}+G_{j}=0, \\
& \left(\frac{\theta f_{j}}{\partial t}\right)_{, e c}=-\frac{1}{\tau_{j}} f_{j}, \frac{1}{\tau_{j}}=\frac{1}{\tau_{f}}\left(\left|C_{j, 1}\right|^{2}+\left|C_{j,-1}\right|^{2}\right)+\frac{1}{\tau_{0}}, \\
& \left(\frac{\partial f_{j}}{\partial t}\right)_{\text {o.f. }}=-\sum_{j j^{\prime} \neq j}\left(\frac{f_{j}}{T_{j^{\prime} j}}-\frac{f_{j^{\prime}}}{T_{j j^{\prime}}}\right) \text {, } \\
& \frac{1}{T_{j^{\prime} j}}=\frac{w_{j^{\prime} j}}{T_{z}} \sum_{m^{\prime} m} \eta_{m^{\prime} m}\left|C_{j m} C_{j^{\prime} m^{\prime}}\right|^{2} \text {, } \\
& w_{j^{\prime} j}=\left\{\begin{array}{ll}
1 & \text { if } E_{j^{\prime}}<E_{j}, \\
\exp \left[-\left(E_{j^{\prime}}-E_{j}\right) / k_{B} T\right\rceil & \text { if } E_{j^{\prime}}>E_{j}
\end{array},\right. \\
& G_{j}=G,\left|\sum_{m} C_{j m} M_{m}\right|^{2}+G_{0}, M_{ \pm 1}=\left(e_{x} \pm i e_{y}\right), M_{ \pm 2}=0 \text {. }
\end{aligned}
$$

Here $\tau_{r}$ and $\tau_{0}$ are the radiative and non-radiative lifetimes introduced in the previous section, $\eta_{m m^{\prime}}=\delta_{\left|m-m^{\prime}\right|, 1}$ or $\delta_{\left|m-m^{\prime}\right|, 3}$ depending on whether the interlevel transitions are connected with apin-flips of an electron or a hole within the exciton. In our calculation we considered the latter possibility. The factor $w_{j}, j$ takes into account the difference in transfer rates for transitions from lower to higher levels and vica versa. For type II apperlattices, the splittings $\left|E_{j r}-E_{j}\right|$ are amall in comparison to the thermal energy $k_{B} T$ even for $T=2 K$ and one can put $w_{j^{\prime} j}=1$. For type I superlattices, the exchange interaction energy is comparable with the liquid helium temperature and the factors $w_{j \prime} j_{j}, w_{j j}$ can noticeably differ from each other. The positive coefficienta $G$, and $G_{0}$ are proportional to the initial light intensity. They describe the generation rates (direct optical excitation or transitions from higher excitonic states) respectively polarization-dependent and -independent ( $e$ is the initial polarization unit vector). Under exactly resonant excitation $G_{0}<G_{\varphi}$, the opposite inequality takes place in the case of non-resonant photoexcitation of free carriers far into the band. For quasi-resonant (or close-toedge) excitation, $G$, and $G_{0}$ are expected to be of the same onder of magnitude. The intensities of the photoluminescence circulurly-polarized components are given by

$$
I_{ \pm} \propto \sum_{j}\left|C_{j, \pm 1}\right|^{2} f_{j}
$$

Note that the above rate equations are valid provided the aplittinga $\left|E_{j^{\prime}}-E_{j}\right|\left(j \neq j^{\prime}\right)$ exceed the energy 
uncertainty characterized by the parameter $\hbar\left(\tau_{0}^{-1}+\tau_{p}^{-1}+\tau_{p}^{-1}\right)$. Otherwise one should apply the exciton apindensity-matrix formalism.

Fig.3 shows the total intensity, $I=I_{+}+I_{-}$, and the degree of circular polarization, $P_{c}=\left(I_{+}-I_{-}\right) / I_{\text {, of }}$ the photoluminescence as a function of the longitudinal magnetic field under anpolarized quasi-resonant optical excitation. The values of parameters used in the calculation are given in the figure caption. Doe to the zero-field splitting $\delta_{2}$ of the radiative doublet (see inset in Fig.3a) two level-anticrossing signals are clearly seen both in lig.3a and Fig.3b. Physically, there are two independent reasons leading to the resonant behaviour of $l$ and $P_{c}$ : (1) the difference between the generation rates, $2\left(G_{p}+G_{0}\right)$ and $2 G_{0}$, to the radiative $(m= \pm 1)$ and non-radiative $(m \pm 2)$ states, (2) the difference between the lifetimes, $\tau_{0} \tau_{r} /\left(\tau_{0}+r_{p}\right)$ and $\tau_{0}$, of these two pairs of states. If the reason 1 is dominant then the anticrossing is manifested by a resonant decrease in intensity and by an apperrance of positive/negative nonthermal circular polarization respectively for the lower and higher radiative levels. On the contrary, if the reason 2 is more important which is the case for the parameter chosen in Fig. 3 one should observe an increasing intensity and negative/positive $P_{0}$ respectively for the lower- and higher-field anticrossings. This is in agreement with the experimental data presented in ${ }^{11}$. Under optical excitation with circularly polarized light, the anticrossing signal is superimposed on the smooth curve $P_{c}(B)$ ariaing due to optical orientation of exciton angular momenta (the inset in Fig.3b). Thas, the derived theory explains the observed ${ }^{11}$ variation of $P_{c}$ with mognetic field and predicts resonant changes in intensity by a few percent.

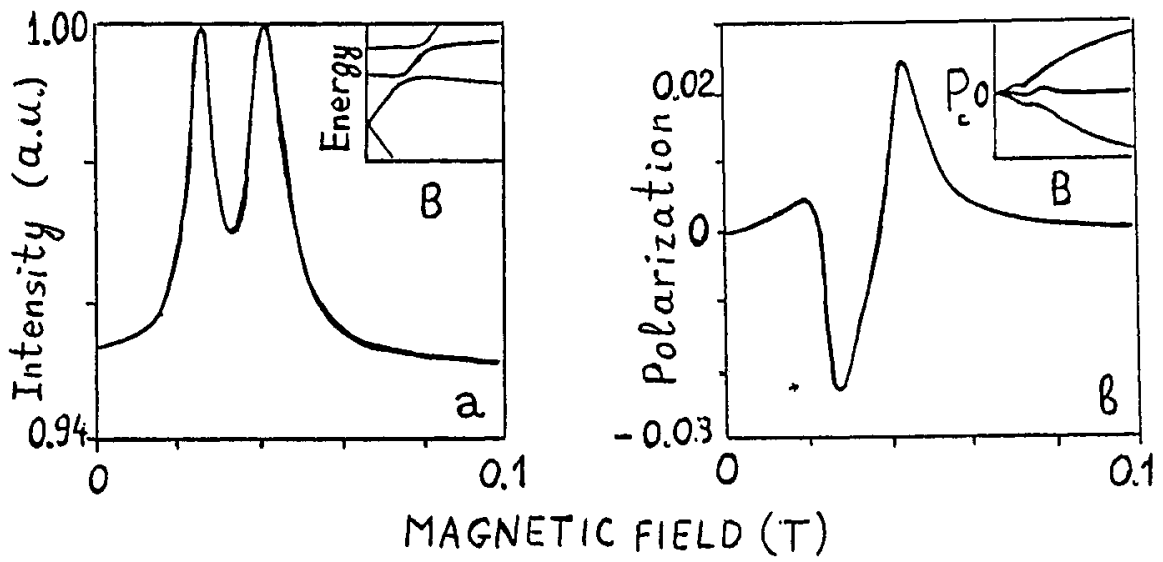

Fig. 3. The calcalated magnetic-field dependences of photoluminescence intensity (a) and circular polarization (b) under quasi-resonant anpolarized optical excitation. The parameters used are: $g_{a}=2.08,3 g_{h}=2.5, \delta_{0}=$ $4.5 \mu \mathrm{eV}, \delta_{2}=2 \mu e V, \delta_{1}=0$ (for the GaAs/AlAs superlattice $17.4 \AA / 26 \AA{ }^{4}$ ), $G,=G_{0}, \tau_{0} / \tau_{,}=4, \tau_{0} / \tau_{0} \gg$ $1,\left|E_{j 1}-E_{j}\right| \ll k_{B} T$ (type II superlattice, the unthermalized quartet). The insets show: (a) the energy-leve! diagram for the heavy-hole exciton and $(b)$ the photoluminescence circular polarization for unpolarized, left $(\sigma-)$ and right $\left(\sigma_{+}\right)$circularly polarized excitation ${ }^{9}$.

\section{RESONANT RAMAN SCATTERING DUE TO ACCEPTOR-BOUND-HOLE SPIN-FLIPS}

In this section we consider spin-polarized excitons acting as intermediate atates in Raman scattering by spin llips of acceptor-bound holes observed in p-type $\mathrm{GaAs} / \mathrm{Al}_{s} \mathrm{Ga}_{1-x}$ As multiple quantum wells ${ }^{12}$. The experiment was performed in the backscattering Faraday configuration $z\left(\sigma_{\eta}, \sigma_{\lambda}\right) z, z$ being parallel to the heterogtructure growth direction and $\eta= \pm, \lambda= \pm$ denoting the circular polarization of the exciting and scattered light respectively. The spin-flip Raman scattering (SFRS) under consideration is related to transitions within the magnetic-field-split ground state of the neutral acceptor and involves the angular-momentum tips $+3 / 2 \rightarrow-3 / 2$ or $-3 / 2 \rightarrow+3 / 2$ of a hole bound to an acceptor. The effect exhibits resonance behaviour and the scattering efficiency is significant unly within a narrow frequency region between the low-energy edge of the photoluminescence spectrum and the fundarnental-absorption edge of the type I heterostructure (Fig.4). The polarization of both the Stokes and antiStokes components was found to depend on the excitation energy, and at least two different mecharisms were identified to contribute to the bound-hole-related SFRS. As well as in the previons sections the following theoretical considerations are based on the selection rules for optical excitation (and emisaion) of el - hhl excitons in the GaAs/AlGaAs quanturn well: trangition to the states $|-1 / 2,3 / 2\rangle$ and $|1 / 2,-3 / 2\rangle$ are allowed respectively for $\sigma_{+}$ and $\sigma_{-}$polarizations and the atates $|1 / 2,3 / 2\rangle$ and $\left.\mid-1 / 2,-3 / 2\right)$ are inactive in the dipole approximation. Here in the notation $(s, m)$ the first and second aymbols indicate the projection of the electron and hole apin on the $z$ axis. 
Fig. 4 Resonance profile of the SFRS efficiency for the $z\left(\sigma_{-}, \sigma_{+}+\sigma_{-}\right) z$ configuration in a magnetic fiedd $B=10 \mathrm{~T}$ (dashed line, foll dots). Photoluminescence spectrum of the maltiple-quantum-wells $48 / 110$ also for $B=10 \mathrm{~T}$ and excitation at $\hbar \omega=1.7 \mathrm{eV}$ (solid line). The circles and dashed-dotted line show the dependence of the circular polarization, $P_{0}$, on the initial phonon energy under circularly-polarized excitation. (From ${ }^{12}$ )

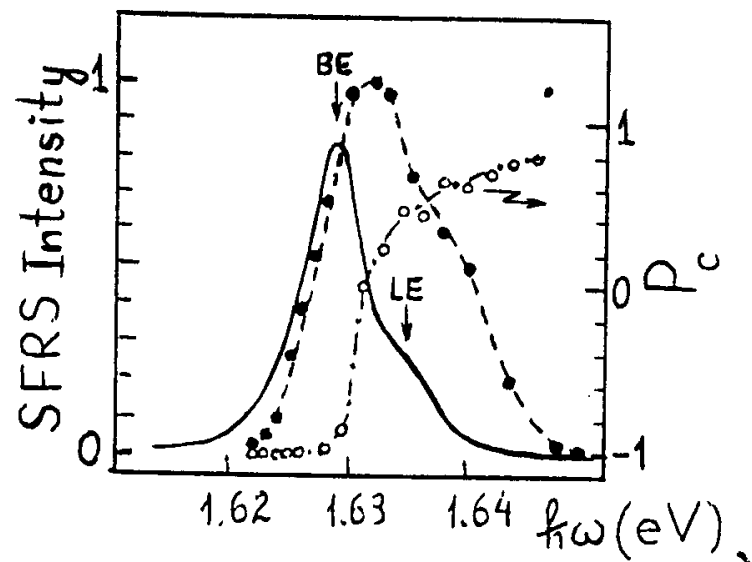

Under excitation at the high-energy edge, both Stokes and anti-Stokes lines are observed in the parallel $z\left(\sigma_{\eta}, \sigma_{\eta}\right) \bar{z}$ configurations with their intensities being independent on the sign of $\eta$. The corresponding scattering process (process $B$ in the rotations of ${ }^{12}$ ) includes three stages: (B1) Absorption of a $\sigma_{\eta}$ photon followed by formation of a three-particle complex consisting of a resonantly photoexcited spiz-polarized exciton $|-\eta 1 / 2, \eta 3 / 2\rangle$ and an equilibrium hole bound to an acceptor neighboring the exciton localization area. (B2) As a result of the hole-hole "flip-stop" interaction

$$
m, m_{A} \rightarrow m,-m_{A}
$$

the acceptor-bound hole changes its sign from $m_{A}= \pm 3 / 2$ to $-m_{A}$ whereas the spin $m$ of the hole in the exciton remains anchanged. (B3) Emission of a $\sigma_{\eta}$ secondary photon by the localized exciton $|-\eta 1 / 2, \eta 3 / 2\rangle$.

The flip-stop interaction operator accounting for the process $B$ can be written as

$$
\mathcal{H}_{S-1}=\sigma_{4}^{h}\left(\Delta_{+} \sigma_{+}^{A}+\Delta_{-} \sigma_{-}^{\Lambda}\right),
$$

where $\Delta_{+}$and $\Delta_{-}$are complex-conjugate coefficients, $\sigma_{ \pm}^{\hat{A}}=\sigma_{x}^{\hat{A}} \pm i \sigma_{y}^{\hat{A}}, \sigma_{\alpha}^{h}$ and $\sigma_{\hat{\beta}}^{A}$ are the analoge of the Paali matrices for a $\pm 3 / 2$ hole in the localized exciton $(h)$ or at the acceptor $(A)$. The operator (17) is similar to the contribution of $J_{x z}^{\prime} S_{i x} S_{j 3}+J_{y z}^{\prime} S_{i y} S_{j}$ to the spin-spin Hamiltonian of the magnetic ions that induces transitions with $\Delta m_{i}= \pm 1, \Delta m_{j}=0$, where $i$ and $j$ are numbers of the lons and $m_{i}, m_{j}$ are the eigenvalues of the spin oyerators $S_{i x}, S_{j y}$. In the axial approximation ${ }^{13}, \Delta_{ \pm}=f(\rho) \rho_{ \pm}^{3}$, where $\rho_{ \pm}=\rho_{x} \pm i \rho_{y}$ and $\left(\rho_{x}, \rho_{y}\right)$ is the twodirnensional vector connecting the acceptor site with the center of localization of the exciton hole. The cubic dependence of $\Delta_{ \pm}$on $\rho_{ \pm}$can be anderstood taking into sccount that in the process $B$ the acceptor-bound-hole changes the $z$ component of its angular momentum by \pm 3 and the photon angular-momentum projection holds anchanged.

At the low-energy edge of the SFRS resonance profile, Stokes and anti-Stokes lines are observed in the crossed $z\left(\sigma_{ \pm}, \sigma_{\mp}\right) \bar{z}$ configurations respectively (process $\mathrm{A}$ in $\left.{ }^{12}\right)$. In the recent paper ${ }^{16}$ it has been unambiguousily shown that excitons bound to neutral acceptors ( $A^{0} X$ complexes) contribate to this scattering. The $A^{0} X$ complexes act as resonant intermediate states and scattering occurs due to an additional acoustic-phonon-assisted spin flip of an electron in the exciton. Thus, the whole scattering process looks as follows. The $\sigma_{\eta}$ circularly polarized light converts $-\eta 3 / 2$ neutral acceptors into $\mid A^{0} X$, a $\rangle$ complexes with the electron uncompensated apin $s=-\eta 1 / 2$ (stage A1). The generation rate is proportional to the equilibrium population, $f_{m}$, of the neutral acceptor state with the apin $m=-\eta 3 / 2$. The spin structure of the Kramers-conjugate states $\left.\mid A^{0} X, s\right)(s= \pm 1 / 2)$ is given by

$$
\left.\mid A^{0} X, s\right)=\left|+\frac{3}{2},-\frac{3}{2} ; s\right\rangle+\frac{3}{4} \frac{\Delta_{h h}}{\Delta_{C}}\left|+\frac{1}{2},-\frac{1}{2} ; s\right\rangle-\frac{\sqrt{3}}{2} \frac{\Delta_{c h}}{\Delta_{C}}|3 s,-s ;-s\rangle .
$$

Here we denote by $\left.\mid m_{1}, m_{2} ; s\right\}$ the state with hole angalar momenta $m_{1}, m_{2}$ and electron spin s, $\Delta_{h h}$ and $\Delta_{\text {eh }}$ are the constants of hole-hole and electron-hole exchange interaction, $\Delta_{C}$ is the zero-magnetic-field splitting between the $\pm 3 / 2$ and $\pm 1 / 2$ neatral scceptor levels due to the quantum confinement effect. While deriving Eq.(18) it was assumed that the constants $\Delta_{h h}$ and $\Delta_{c h}$ are small as compared with the value of $\Delta_{C}$. The next stage A2 is the spin-flip $\left|A^{0} X, s\right\rangle \rightarrow\left|A^{0} X,-s\right\rangle$ due to sbsorption or emission of an acoustic phonon. The most probable mechanism of the spin-lattice relaxation in $A^{0} \mathrm{X}$ complexes is determined by the admixture of the $|3 s,-s ;-s|$ state in Eq.(18). At the final stage, $A 3$, the $A^{0} X$ complex annihilates with the emission of a $\sigma_{-\eta}$ photon and the remaining bound-hole finds itself in the inverted state $\eta 3 / 2$. It follows then that, for a positive hole $g$-factor and in a magnetic field directed along the $z$ axis, the Stokes scattering occurs in the $z\left(\sigma_{+}, \sigma_{-}\right) z$ configuration and the anti-Stokes line should be present only in the $z\left(\sigma_{-}, \sigma_{+}\right) \bar{z}$ apectrum, in agreement with the experiment.

Another important consequence is that, in contrast to the process $B$ where the Raman ahift is given by $\Delta E=$ $3 g_{A} \mu_{0} B_{3}$ ( $g_{A}$ is the acceptor-bound hole g-factor), the shift for the process $A$ includes the Zeeman splitting of the 
electron spin atates in the $\mathrm{A}^{0} \mathrm{X}$ complex: $\Delta E=\left(3 g_{A}-g_{6}^{\prime \prime}\right) \mu_{0} B_{k}$. This has been verified in experiments ${ }^{14}$ at a tilted magnetic field in which, in addition to the acoustic-phonon-assisted scattering, no-phonon SFRS becomes allowed aince the electron in the $\mathrm{A}^{0} \mathrm{X}$ complex can change its apin due to Zeeman interaction with the in-plane field component. The main result of measurements at tilted magnetic fields is the appearance of an additional Raman line under resonant excitation of $A^{0} \mathrm{X}$ complexes. Thus the Raman technique enables one to measure directly both the neatral-acceptor and electron 8 -factors as a function of the quantum well width. For the $\mathrm{GaAs} / \mathrm{Al}_{0.33} \mathrm{Gr}_{0.67} \mathrm{As}$ multiple-quantum-well structures with the layer thicknesses $46 / 110 \AA, 72 / 110 \AA$ and $102 / 110 \AA$ the following values of the g-factors has been found ${ }^{14}: 3 g_{A}=2.4 ; 2.18 ; 2.09$ and $g_{l}^{\|}=0 ;-0.11 ;-0.23$.

In addition to the strongest $\pm 3 / 2 \rightarrow \mp 3 / 2$ SFRS lines Sapega et al. ${ }^{14}$ have observed two other lines. Their polarization properties and magnetic-field behavior indicate that they originate from the $+3 / 2 \rightarrow-1 / 2$ and $-3 / 2 \rightarrow$ $1 / 2$ interlevel transitions with the Raman shift

$$
\Delta( \pm 3 / 2 \rightarrow \mp 1 / 2)=\Delta_{C} \mp g_{A} \mu_{0} B\left(\frac{1}{2} \sqrt{\cos ^{2} \phi+4 \sin ^{2} \phi}+\frac{3}{2} \cos \phi\right),
$$

where $\phi$ is the angle between $\mathbf{B}$ and $z$. The interlevel acattering can be described taking into account the hole-hole exchange interaction in the $A^{0} X$ complex and an admixture of $\pm 1 / 2$ holes in the ground state presented by Eq.(18). For the above three samples the measured values of the 'crystal-field' splitting $\Delta_{C}$ are equal respectively to 7.3 ; 3.5 and $2 \mathrm{meV}^{14}$.

\section{OPTICAL ORIENTA'TION OF HOT EXCITONS UNDER DOUBLE 2s-1s RESONANCE CONDITIONS}

Optical orientation methods can be helpful to analyze mechanisms of doubly-resonant secondary emission of semiconductors. In optical spectroscopy the double resonance is defined as enhancement in the intensity of secondary emission ander conditions where (i) the energies, $\hbar \omega$ and $\hbar \omega \prime$, of the incident and secondary photons coincide with those of two interband excitations (excitons) and (ii) the difference $\hbar\left(\omega-\omega^{\prime}\right.$ ) equals to the energy of one or few optical phonons. This effect which can be described in terms of both resonant Raman scattering and resonant photoluminescence has been observed in bulk materials (see ${ }^{16}$ and references therein) as well as in quantura-well structures ${ }^{16,17}$.

In ${ }^{16,17}$ resonant states for incoming and outgoing channels were $1 \mathrm{~s}$-excitons attached to different size-quantized sabbands or to different Landan levels. In ${ }^{3}$ the double optical resonance has been observed on the states $e 1-h h 1(2 s)$ and $e 1-h h 1(1 s)$ belonging to the same excitonic series and related to the lowest electron and hole subbands. CdTebased quantum well srtuctures were chosen for the observation of the 2a-1s resonance because of the following ressons: (1) In CdTe, the energy of a longitudinal optical (LO) phonon is relstively low: $\hbar \Omega_{L O}=21 \mathrm{meV}$, (2) In quantum wells $\mathrm{CdTe} / \mathrm{Cd}_{1-s} \mathrm{Mn}_{3} \mathrm{Te}$ (or $\mathrm{CdTe} / \mathrm{Cd}_{1-s} \mathrm{Mgs}_{s} \mathrm{Te}$ ) the energy difference $\hbar \omega_{21}$ between the $2 \mathrm{~s}$ and 18 exciton levels remarkably increases as a result of the quantum confinement effect and differs from $k \Omega_{L O}$ jnat by a few meV. (3) In the magnetic field oriented along the growth axis, $z$, the 2s exciton level andergoes a strong diamagnetic blue shift and the condition $\omega_{21}=\Omega_{L O}$ is fulfilled in a moderate field $B<10 T$. The dorble $28-1 \mathrm{~s}$ resonance has been observed in backscattering Faraday geometry for circularly polarized analyzer and polarizer. In both photoluminescence (PL) and photoluminescence excitation (PLE) spectra the intensity of the sharp 1LOreplica has been found to increase rapidly with tuning to the donble resonance conditions (Fig.5). An important fact is that a strong 1LO-line has been observed not only in parallel circular polarizations but also in the crossed $z\left(\sigma_{+}, \sigma_{-}\right) \bar{z}$ or $z\left(\sigma_{-}, \sigma_{+}\right) \bar{z}$ configuration.

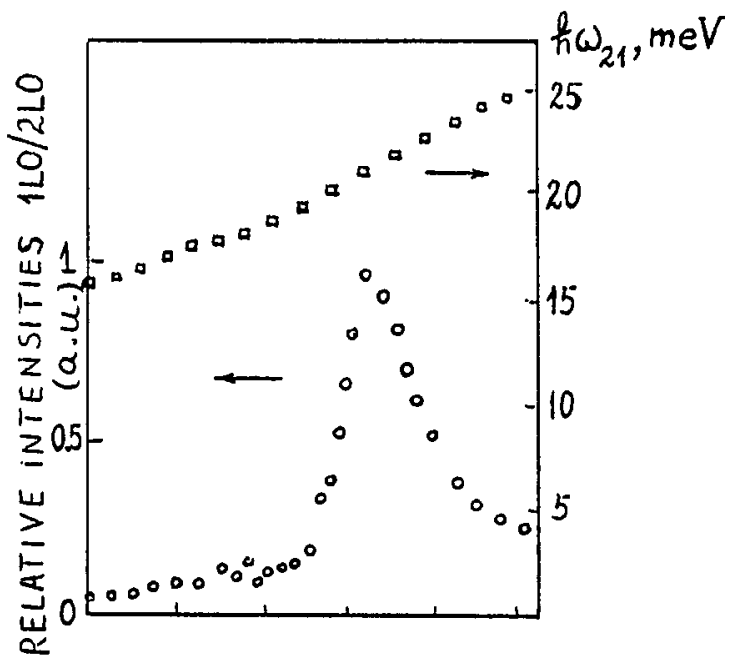

Fig. 5 The magnetic field dependence (circles) of the ratio between intensities of the $1 \mathrm{LO}$. and $2 \mathrm{LO}$ - lines under double 2s-1a resonance observed in the $\mathrm{CdTe} / \mathrm{Cd}_{0.80} \mathrm{Mn}_{0.14} \mathrm{Te} 85 \dot{A} / 95 \AA$ multiple-quantum-well structure. Squares show the 2a-1s energy distance as a function of the 'magnetic field. (From ${ }^{3}$ ) 

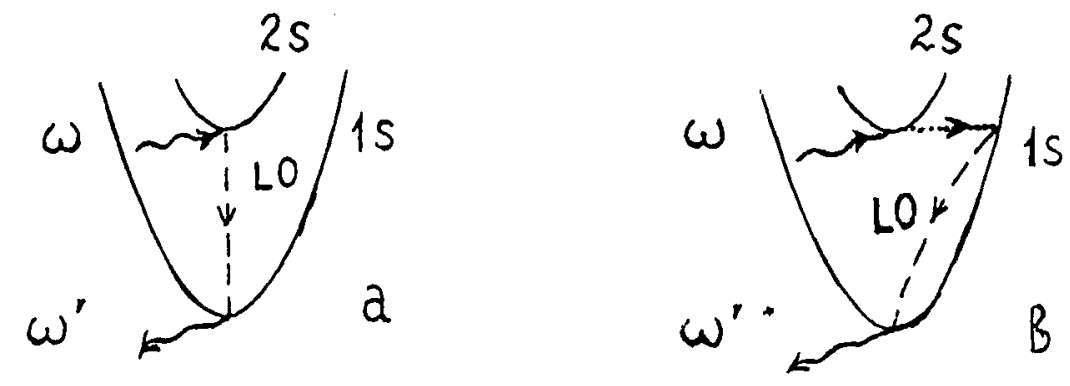

Fig. 6 Schematic representation of two possible mechaniams for doubly-resonant 28-1s 1LO-ussiated secondury emission.

Let us consider two possible mechanisms of the donbly-resonant 28-18 secondary emission. The both are illuatrated in Fig.6. For the direct doubly-resonant one-phonon Raman scattering (see the two-step process of Fig.6a), optical excitation of the 28 exciton is followed by the LO-phonon-assiated transition to the bottom of the 18-exciton subband and then by emission of a photon from the 18 state. However the analysis shows that the vertical transition $2 a-1+L O$ is ineffective. Really, in a bulk semiconductor, such a transition is forbidden in

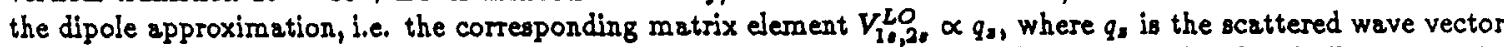
(the difference between wave vectors of the incident and aecondary photons). Remind that by similar resson in resonant spectrs of secondary emission of perfect crystals the 1LO-line is much wealser than the $2 L O$ line. On the other hand, in the two-dimentional approximation the exciton envelope function is factorized so that

$$
\Psi_{n,}=f_{n}(\rho) \varphi_{a !}\left(z_{0}\right) \varphi_{h_{1}}\left(z_{h}\right)
$$

with the some single electron and hole envelopes $\varphi_{a 1}, \varphi_{h 1}$ for 18 and 28 states. Here $f_{x,}(\rho)$ is the envelope fnnction describing the relative electron-hole motion in the $(x, y)$ plane and $\rho=\left[\left(x_{e}-x_{h}\right)^{2}+\left(y_{b}-y_{h}\right)^{2}\right]^{1 / 2}$. The condition of orthogoinality between $f_{1},(\rho)$ and $f_{2,}(p)$ immediately makes zero the matrix element $V_{1 s, 2,}^{L O}$ aince for backscattering of normally-incident light the exciton-phonon interaction operator is $\rho$-independent. Thus, the value of $V_{1,20}^{L}$, can be nonvanishing only due to the Coulomb-potential effect apon the electron or hole confinement along the $z$ direction. Furthermore, for the direct process of Fig.68 the exciton polarization cannot andergo a remarkable change which contradicts to the presence of the strong 1LO. Ine in the apectra observed in the crossed circular polarizations.

The more realistic mechanimm is illostrated in Fig.6b: the 28-exciton is first acattered from the bottom of the 28 branch to the "hot" is atates characterized by large in-plane center-of-mass wave vectors, $\mathbf{K}$, then it is multiscattered by static random potential of the heterostructure and only afterwards emito a LO-phonon. Obvionsly the second mechanism has more grounds to be interpreted as a lominescence rather than Raman scattering. It should be mentioned that similar static scattering processes with a large momentum transfer were snggested by Kleinman et al..$^{16}$ and Gubarev et al..$^{36}$ in order to interpret their resolts on doubly resonant LO-phonon-asaiated secondary emission respectively in $\mathrm{GaAs} / \mathrm{AJ}_{8} \mathrm{Ga}_{1-8} \mathrm{As}$ quantum wells and balk semimagnetic semiconductor $\mathrm{Cd}_{0.96} \mathrm{Mn}_{0.05} \mathrm{Te}$.

According to the theory of resonant secondary emisaion in semiconductors (see ${ }^{1}$ ), for the process shown in Fig.6b the spectral intensity, $I\left(\omega^{\prime}, \omega\right)$, of the 1 LO-phonon replica can be presented in the form

$$
I\left(\omega^{\prime}, \omega\right) \propto \delta\left(\omega^{\prime}-\omega+\Omega_{L O}\right) \sum_{\mathbf{K}_{f f^{\prime}}} s_{f f^{\prime}}\left(\mathbf{K}, \omega^{\prime}\right) F_{f f^{\prime}}(\mathbf{K}),
$$

where

$$
S_{f^{\prime}}(\mathbf{K}) \propto g_{1 / m^{\prime}}\left(\omega^{\prime}\right) \sum_{q}\left(\|\left. M_{1 / m^{\prime}}\left(\mathbf{e}^{\prime}\right)\right|^{2} \tau_{1 l m^{\prime}} V_{1 l m^{\prime}, \mathbf{K} f}^{L O, q} V_{1 / m^{\prime}, \mathbf{K} f^{\prime}}^{L O, q}\right)
$$

and $F_{f f^{\prime}}(\mathbf{K})$ is the hot 1s-exciton apin-density matrix which obeys the kinetic equation

$$
\left[\frac{1}{\tau_{t}}+i\left(\omega_{\mathbf{K}_{f}}-\omega_{\mathbf{K}}\right)\right] F_{f^{\prime}}(\mathbf{K})+\sum_{\mathbf{K}} w_{\mathbf{K}} \mathbf{K}\left[F_{f f^{\prime}}(\mathbf{K})-F_{f^{\prime}}\left(\mathbf{K}^{\prime}\right)\right]=G_{f^{\prime \prime}}(\mathbf{K})
$$

with the generation matrix given by

$$
G_{f f^{\prime}}(\mathbf{K})=C J_{0}\left|M_{2,, m}(\mathbf{e})\right|^{2} \Delta\left(\omega_{2 s, m}-\omega, \Gamma_{2}\right) \tau_{2} \frac{2 \pi}{\hbar^{2}} D_{\mathbf{K}_{f, 0 m}^{1,2,}}^{1,18,20 *} \delta\left(\omega_{1} \mathbf{K}-\omega\right) .
$$

The notations used are as follows: $w_{1} J_{0}$ and $e$ are the incident light frequency, intensity and polarization anit 
vector, $\omega^{\prime}$ and $\mathrm{e}^{\prime}$ refer to the secondary radiation,

$$
\Delta\left(\omega_{2,, m}-\omega, \Gamma_{2}\right)=\frac{1}{\pi} \frac{\Gamma_{2}}{\left(\omega_{2 r, m}-\omega\right)^{2}+\Gamma_{2}^{2}}
$$

$\omega_{2 a, m}$ and $\Gamma_{2}$ are the resonance frequency and homogeneous broadening of the 2s-exciton, $m$ is the exciton apin index. $D_{\mathbf{K}, 0 m}^{1 t, 2 s}$ is the matrix element of elastic scattering from the state $2 s, K=0$ to the hot state $1 s, \mathbf{K} f$ with the energy $h \omega_{1} K_{f}$ and two-dimensional wave vector $\mathbf{K}$, the index $f$ enumerates the hot-exciton sublevels. The 2s-exciton lifetime $\tau_{2}$ is connected with $\Gamma_{2}$ and $D^{10,21}$ by the relations

$$
\frac{1}{r_{2}}=2 \Gamma_{2}=\frac{2 \pi}{\hbar^{2}} \sum_{\mathbf{K} /}\left|D_{\mathbf{K}, 1,0 m}^{10,2,}\right|^{2} \delta\left(\omega_{1} \mathbf{K}_{f}-\omega_{2 \varepsilon, m}\right) .
$$

${ }^{w_{\mathbf{K}}} \mathbf{K} \mathbf{K}$ is the probability rate for the elastic scattering $\mathbf{K} \rightarrow \mathbf{K}^{\prime}, \tau_{\epsilon}$ is the hot exciton lifetime with respect to inelastic processes, and $V_{1 l m^{\prime}, \mathbf{K}_{f}}^{L O}$ is the matrix element for the transition $1 \mathrm{~s}, \mathbf{K} f \rightarrow 1 / \mathrm{m}^{\prime}$ accompanied by the emission of the LO-phonon with the wave vector $q$. For aimplicity we neglect the LO-phonon bulk dispersion and confinement energy and consider the phonon frequency $\Omega_{L O}$ as a constant. The index $/$ enumerates the emitting weakly-localized and extended 18 -excitor states with the spin $m^{\prime}= \pm 1$ and lifetime $T_{1 / m^{\prime}}, g_{11 m^{\prime}}\left(\omega^{\prime}\right)$ is the density of sach states, the angle brackets in $\mathrm{Eq}_{\mathrm{q}}$ (21) indicate averaging over different states with the asme excitation energy $\hbar \omega^{\prime} . M_{2,, m}(\mathrm{e})$ and $M_{1 / m^{\prime}}\left(\mathbf{e}^{\prime}\right)$ are the matrix elements of the exciton-photon interaction. Since here we consider backscattering of normally-incident polarized light in the parallel or crossed circular configurations $M_{2 s, m}(e)$ is non-zero for $m= \pm 1$ respectively under $\sigma_{+}$or $\sigma_{-}$excitation and similarly $M_{2, m^{\prime}}(e)$ is non-zero for $m^{\prime}$ corresponding to the detection polarization $\sigma_{ \pm}$.

If the exciton energy $\hbar \omega_{1} \mathbf{K}_{t}$ is spin-independent and spin-flip processes are neglected, then the polarization of the 1LO-line coincides with the initial light circular polarization. However, due to the long-range electron-hole exchange interaction, the exciton states $1 \mathbf{K} f$ longitudinal and tranoverae with respect to the wave vector $\mathbf{K}$ are aplit with the splitting $\hbar \tilde{\Omega}(K)$ vanishing linearly with $K$ as $K \rightarrow 0^{19}$. This results in the effective exciton spin relaxation described by the inverse spin-relaxation time ${ }^{10}$

$$
\frac{1}{T_{l}}=\bar{\Omega}^{2}(K) \frac{\tau^{*}}{1+\left(\Omega_{0} \tau^{*}\right)^{2}}
$$

where $\hbar \Omega_{0}$ is the magnetic-field-indaced aplitting between the exciton atates with $m=1$ and $m=-1$ and $\tau^{*}$ is the momentum scattering time. Note that the bulk-exciton apin-relaxation due to the longitudinaj-transverse splitting was considered by Pikus and Ivchenkot.

If the apin-relaxation time $T_{r}$ is much amaller than the hot-exciton inelastic acattering time $\tau_{0}$ the 1 LO-line polarization is insensitive to the initial polarization while its intensity can depend on e via the factor $\left|M_{21, m}(e)\right|^{2}$ in Eq. (23). Thas the observation of a remarkable 1LO-replica in the crossed $\left(\sigma_{+}, \sigma_{-}\right)$or $\left(\sigma_{-}, \sigma_{+}\right)$configuration is readily understood provided $T_{s} \leq \tau_{s}$.

The integral intensity $J(\omega)=\int d \omega^{\prime} J\left(\omega^{\prime}, \omega\right)$ and integral photoexcitation intensity $J_{P E}\left(\omega^{\prime}\right)=\int d \omega I\left(\omega^{\prime}, \omega\right)$ wre given by Eq.(20) where the $\delta$-function is removed, $\omega$ and $\omega^{\prime}$ aatisfy the relation $\omega^{\prime}=\omega-\Omega_{L O}$ and either $\omega$ or $\omega^{\prime}$ is considered as a varying parameter. The frequency dependences $J\left(\omega^{\prime}\right), J_{P E}(\omega)$ are determined by the function $S_{f \mu}\left(K, \omega-\Omega_{L O}\right)$ in Eq.(20), by the ameared $\delta$-function in Eq. (23) and by inhomogeneous broadening. Qualitative analysis of the behavious of $g_{11 m^{\prime}}\left(\omega^{\prime}\right), r_{1 / m^{\prime}}$ and $\left|M_{1 / m^{\prime}}\left(\mathrm{e}^{\prime}\right)\right|^{2}$ near the exciton band bottom $\omega_{10, m^{\prime}}$ ahows that $S_{f f}\left(K, \omega^{\prime}\right)$ shonld exhibit a sharp peak red-shifted from $\omega_{1,, m^{\prime}}$ by $\delta \omega \approx \Delta(P L-P L E)$ and characterized by the width $2 \gamma \leq \Delta(P L-P L E)$. Here $\Delta(P L-P L E)$ is the Stokes shift between the PL peak excited in off-resonant conditions and the peak in the PLE spectram taken st some frequency below the 18 free-exciton resonance (for the stadied samples $\Delta(P L-P L E) \approx 0.2 \mathrm{meV})$. Furthermore, let $P\left(\omega_{10, m}-\bar{\omega}_{10, m}\right)$ be the statistical distribution of the frequency $\dot{\omega}_{1 s, m}$ with maximum at the point $\bar{\omega}_{10, m}$ and with the characteristic width $2 \bar{\Gamma}$. If $\gamma<\Gamma_{2}, \bar{\Gamma}$ and fluctuations of the energy distance between the exciton levels 28 and 18 are negligible as compared with $\Gamma_{2}$ and $\tilde{\Gamma}$ then the dependences of $J_{P E}\left(\omega^{\prime}\right)$ for the 1LO-phonon replica upon the frequency $\omega^{\prime}$ and $28-18$ spacing, $\omega_{21}$, are governed respectively by the parameters $\bar{\Gamma}$ and $\Gamma_{2}$ through the factor $P\left(\omega^{\prime}-\bar{\omega}_{1, m^{\prime}}\right)$ and $\Delta\left(\omega_{21}+\delta \omega-\Omega_{L O}, \Gamma_{2}\right)$. Thus, from the resonance profile of Fig.5 one can find $2 \hbar \Gamma_{2}=2.6 \mathrm{meV}$ or $\hbar \Gamma_{2}=1.3 \mathrm{meV}$ for homogeneons broadening of the 2s-exciton resonance. The inhomogeneous broadening found from the width of the e1-hh1(18) peak in the PLE spectrum equals to $2 \hbar \bar{\Gamma}=2.1 \mathrm{meV}$ and is comparable with $2 \hbar \Gamma_{2}$.

\section{CONCLUSION}

Thus, the optical spectroscopy of apin-polarized excitons is an effective tool to propose and investigate microscopic mechanisms of the observed optical processes and to measure the exciton fine-structure parameters and damping rate as well as electron and hole g-factors.

The anthor is gratefol to P.Lavallard, A.Yu.Kaminskii, D.N.Mirlin and D.R. Yakoviev for useful discussions. 


\section{REFERENCES}

1. G.E.Pikus, E.L.Ivchenko, "Optical orientation and polarized luminescence of excitons in semiconductors", In: Excitons, ed. by E.I.Rashba and M.D.Sturge, North-Holland, 1982, pp.205-266.

2. W.M.Chen, M.Godlewsld, B.Monemar, J.P.Bergman, "Steady-statelevel-anticrossing for bound-exciton triplets associated with complex defects in semiconductors", Phys. Rev. B, vol. 41, pp. 5746-5755, 1980.

3. D.R. Yakovlev, W.Osaan, A.Waag, G.Landwehr. "Double 2s-1s optical resonance in quantum-well structures", To be published.

4. C.Gourdon, P.Lavallard, "Fine atructure of heavy excitons in GaAs/AlAs anperlattices", Phys. Rev. B, vol. 46, Pp. 4644-4650, 1992.

5. E.L.Ivchenko, V.P.Kochereshko, A.Yu.Naumov, I.N.Uraltsev, P.Lavallard, "Magnetic-field-effecta on photolaminescence polarization in type II GaAs/AlAs superlattices", Superlatt. Mieroatruct., vol. 10, pp. 497-501, 1991.

6. H.W. van Kesteren, E.C.Cosman, W.A.J.A. van der Poel, C.T.Foxon, "Fine structure of excitons in type-II GaAs/AlAs quantom wells", Phys. Rev. B, vol. 41, pp. 5283-5292, 1990.

7. E.L.Ivchenko, A.Yu.Kamingkil, LL.Aleiner, "Exchange spliting of excitonic levels in type I and II superiattices", JETP, vol. 77, pp. 609-616, 1993.

8. G.Edwards, J.C.Inkson, "Hole states in GaAs/AlAs heterostructures and the limitations of the Luttinger model", Solid State Commun., vol. 89, pp. 595-599, 1994.

9. E.L.Ivchenko, A.Yu.Kaminskii, "Optically detected anticrossing of excitonic levels in superlattices in a masnetic field" , In: Nanostructurea: Physica and Technology, Proc. Int. Symposium, St.Petersburg, Russia, 1994, pp. 58-61.

10. C.Gourdon, D.Yu.Rodichev, P.Lavallard, G.Bacquet, R.Planel, "Anisotropic exciton states in GaAs/AlAs superiattices in zero and non-zero magnetic field", J. de Physique IY, vol. 3, Suppl. JPII, C5, pp. 183-186, 1993.

11. P.G.Baranov, I.V.Mashkov, N.G.Romanov, P.Lavallard, R.Planel, "Optically detected magnetic resonance of excitons and carriers in psendodirect GaAs/A]As superiattices", Solid State Commun., vol. 87, pp. 649-654, 1993.

12. V.F.Sapega, M.Cardona, K.Ploog, E.L.Ivchenko, D.N.Mirlin, "Spin-flip Raman scattering in $\mathrm{GaAs} / \mathrm{Al}_{2} \mathrm{Ga}_{1} \rightarrow \mathrm{As}$ maltiple quantum wells", Phys. Rev. B, vol. 45, pp. 4320-4326, 1992.

13. E.L.Ivchenko, "Exchange interaction and scattering of light with reversal of the hole angular momentum at an acceptor in quantum-well structures", Sov. Phys. Solid State, vol. 34, pp. 254-260, 1992.

14. V.F.Sapega, T.Ruf, M.Cardona, K.Ploog, E.L.Ivchenko, D.N.Mirlin, "Resonant Raman scattering due to bonnd-carrier spin flip in $\mathrm{GrAB} / \mathrm{Al}_{s} \mathrm{Ga}_{1-2} \mathrm{As}$ quantum wells", Phys. Rev. B, vol. 50, $15 \mathrm{Jdy}, 1994$.

15. S.I.Gubarev, T.Ruf, M.Cardona, "Doubly resonant Raman scattering in the semimagnetic semicondactor $\mathrm{Cd}_{0.96} \mathrm{Mn}_{0.05} \mathrm{Te}^{n}$, Phys. Rev. B, vol. 43, pp. 1551-1554, 1981.

16. D.A.Kleinman, R.C.Miller, A.C.Gossard, "Doubly resonant LO-phonon Raman scattering observed with GaAs-Al, $\mathrm{Ga}_{1-2} \mathrm{As}$ quantum wells", Phys. Reu. B, vol.35, pp. 664-674, 1987.

17. F. Calle, J.M. Calleja, F. Meseguer, C. Tejedor, L. Vina, C. Lopez, C. Ploog, "Donble Raman resonances indaced by a magnetic field in GaAs-AlAs multiple quantam wells", Phys. Rev. B, vol. 44, pp. 1113-1117, 1991.

18. L.C.Andreani, F.Basaani, "Exchange interaction and polariton effects in quantum-well excitons", Phys. Rev. $B$, vol. 41, pp. 7536-7544, 1890.

19. M.Z.Maialle, E.A. de Andrada e Silva, L.J.Shrm, "Exciton spin dinamics in quantum wells", Phys. Rev. B, vol. 47, pp. 15776-15788, 1883. 